\title{
Amélioration de la germination des faînes (Fagus silvatica) par prétraitement en présence de polyéthylène glycol
}

\author{
Claudine MULLER et M. BONNET-MASIMBERT \\ I.N.R.A., Station d'Amélioration des Arbres forestiers \\ Centre de Recherches forestières, Champenoux, F 54280 Seichamps \\ * I.N.R.A., Station d'Amélioration des Arbres forestiers \\ Centre de Recherches d'Orléans, Ardon, F 45160 Olivet
}

\begin{abstract}
Résumé
Pour la levée de dormance des faînes, une préréfrigération sans milieu semble meilleure que la stratification classique en tourbe humide, mais elle nécessite le maintien, tout au long de ce traitement, d'une teneur en eau des faînes voisine de 30 p. 100. L'installation des faînes sur une solution contenant 150 à $200 \mathrm{~g}$ de polyéthylène glycol (PEG 6000 ) par kilo assure ce maintien et évite ainsi toute sortie de radicule au cours du passage au froid. De plus, lorsque la durée de celui-ci est insuffisante, cette méthode permet une augmentation simultanée du taux et de la vitesse de germination. Il s'agit là de résultats préliminaires encourageants, mais l'utilisation pratique d'une telle technique nécessitera encore toute une mise au point.
\end{abstract}

\section{Introduction}

Les faînes sont affectées d'une dormance embryonnaire, très variable d'une faîne à b'autre, et d'un lot à l'autre, qui nécessite pour être levée un prétraitement au froid humide pouvant durer de 1 à 3 mois. Semées sans prétraitement, leur germination est très faible et étalée dans le temps. Cette grande hétérogénéité de degré de dormance a orienté les recherches menées ces dernières années vers l'accélération et la synchronisation de la germination (Muller, 1977, 1982; Muller \& Bonnet-Masimbert, 1981 ; SuszKa, 1979 ; SuszKa \& KluCZYNSKA, 1980), notamment par substitution d'une technique de préréfrigération sans milieu à la classique stratification en milieu humide. Dans cette technique récemment mise au point sur faînes par Suszka (1979), la levée de dormance s'effectue à $3{ }^{\prime \prime} \mathrm{C}$, avec un taux d'humidité de 28-30 p. 100 pendant une durée supérieure de 2 semaines environ à celle de la stratification classique. Grâce à cette teneur en eau contrôlée, on évite la germination qui affecte certaines faînes lors d'une stratification classique. 
Or, dans le domaine agricole, on applique de plus en plus fréquemment, sous le nom de «priming», un prétraitement des semences en présence d'agents osmotiques, pour améliorer les rendements et synchroniser les levées. Les agents osmotiques utilisés peuvent être du chlorure de sodium ou des glucides (mannitol), mais surtout, actuellement des solutions aqueuses de polyéthylène glycol (PEG), substance considérée comme chimiquement inerte, en particulier sous la forme de «Carbowax 6000 » [Parmer \& Moore, 1968 (maïs), Kaufmann, 1969 (laitue), Kaufmann \& Ross, 1970 (laitue, blé), Heydecker et al., 1973 (carottes, betteraves), Mac DonOugh, 1976 (graminées fourragères)], puis bien daautres. Sur semences forestières, les essais sont moins nombreux bien que parfois assez anciens et ne concernent que des gymnospermes [SAToo \& Goo, 1954 (Cryptomeria japonica, Chamaecyparis obtusa, Pinus densiflora), Larson \& Schubert, 1969 (Pinus ponderosa), Djavanshir \& ReID, 1975 (Pinus eldarica, $P$. ponderosa), Simak, 1976 ( $P$. sylvestris)].

La technique développée par Heydecker (1974) consiste à placer les semences au contact d'une solution de PEG. Par le contróle de la concentration de la solution, de la température et de la durée du traitement, on réalise une imbibition partielle de la graine, suffisante pour permettre le déroulement des processus prégerminatifs, mais insuffisante pour assurer l'émergence de la radicule, donc la germination.

Ainsi, un traitement suffisamment long peut permettre la levée de dormance de l'ensemble des graines d'un lot hétérogène quant à son degré de dormance, sans risque de germination. Dès que les graines sont soustraites à l'influence de l'agent osmotique et placées en condition de germination, celle-ci se produit de manière très groupée. Par ailleurs, les meilleurs résultats de prétraitements osmotiques sont obtenus lorsqu'ils sont effectués à température relativement basse $\left(10\right.$ à $15^{\circ} \mathrm{C}$ ) (HeYdecker, 1974 ; SALTER \& Darby, 1976 ; SimaK, 1976).

Nous avons donc entrepris en 1981 des essais de prétraitement osmotique à basse température sur faînes (Fagus silvatica) qui font l'objet de cette note préliminaire.

\section{Matériel et méthode}

Deux essais ont été réalisés : l’un porte sur l'élude de la germination à la suite d'un traitement de PEG et concerne un lot récolté en 1980 en forêt de Halatte; l'autre porte sur l'étude de l'imbibition des faînes en présence de PEG et, faute de disposer de suffisamment de faînes du premier lot, concerne un lot récolté en 1980 en forêt de Compiègne.

\subsection{Essai sur la germination}

Les faînes sont placées en boîtes de Pétri $(\varnothing 14 \mathrm{~cm})$ sur papier filtre imprégné d'eau (témoin) ou de solutions de PEG (Carbowax 6000 ) à différentes concentrations. Les pressions osmotiques correspondantes ont été estimées d'après la méthode de Williams \& ShaYKeWiCH, 1969. Le prétraitement est effectué à +3 "C, température optimale pour la levée de dormance des faînes. On compare deux durées de prétraitement : 6 et 8 semaines. Il y a 150 faînes par concentration de PEG et par durée. La durée de 6 semaines $(\mathrm{x})$ correspond au temps nécessaire à l'obtention de 10 p. 100 de 
faînes germées en milieu humide à +3 "C (Suszka \& ZiETA, 1976). C'est la durée optimale de la stratification classique. La durée 8 semaines $(x+2)$ correspond à la durée optimale de prétraitement sans milieu (préréfrigération). Après traitement, les faînes sont lavées à l'eau courante, ressuyées en surface quelques minutes, puis mises à germer en boîte de Pétri sur eau, en température alternée $5^{\circ} \mathrm{C}(14 \mathrm{~h}), 15^{\circ} \mathrm{C}(10 \mathrm{~h})$.

\subsection{Essai sur l'imbibition}

A la suite de l'essai précédent, nous avons voulu suivre le processus de réimbibition selon la concentration en PEG. Les conditions du prétraitement sont les mêmes que ci-dessus. Après $1,2,4,6,8$ et 10 semaines à +3 "C on prélève, pour chaque concentration de PEG, 50 faînes sur lesquelles on procède à une mesure de teneur en eau par séchage à $105^{\circ} \mathrm{C}$ pendant 24 heures. Les résultats sont exprimés par rapport au poids frais.

\section{Résultats}

\subsection{Essai sur la germination}

L'influence de la durée du prétraitement et de la concentration en PEG apparaît sur le tableau 1, les courbes de germination pour chaque traitement étant regroupées

\section{TABLEaU 1}

Influence d'un prétraitement osmotique sur polyéthylène glycol, sur le taux et la vitesse de germination des faînes.

The influence of an osmotic treatment with PEG, on the rate and the speed of the germination.

\begin{tabular}{c|c|c|c|c|c}
\hline $\begin{array}{c}\text { Durée du } \\
\text { prétraitement } \\
\text { (semaines) }\end{array}$ & $\begin{array}{c}\text { Concentration } \\
\text { en PEG } \\
\text { (en g/kg) }\end{array}$ & $\begin{array}{c}\text { Pression } \\
\text { osmotique } \\
\text { (bar) }(1)\end{array}$ & $\begin{array}{c}\text { \% de pré- } \\
\text { germination } \\
\text { durant le } \\
\text { prétraitement }\end{array}$ & $\begin{array}{c}\text { Taux de } \\
\text { germination } \\
\%\end{array}$ & $\begin{array}{c}\text { Temps moyen } \\
\text { de germination } \\
\text { (j) (2) }\end{array}$ \\
\hline 6 & 0 & témoin & 3,3 & $72,0^{\mathrm{a}}$ & 33,4 \\
$(\mathrm{x})$ & 100 & $-1,5$ & 1,3 & $67,3 \mathrm{ab}$ & 46,6 \\
& 150 & $-3,5$ & 0,7 & $80,7 \mathrm{~b}$ & 23,2 \\
& 208 & -8 & 0 & $74,7 \mathrm{ab}$ & 19,3 \\
\hline & 0 & témoin & 25,3 & $80,0^{\mathrm{b}}$ & 15,6 \\
$(\mathrm{x}+2)$ & 100 & $-1,5$ & 17,3 & $83,3 \mathrm{~b}$ & 15,7 \\
\hline
\end{tabular}

(1) Estimation de la pression osmotique d'après Williams et ShayKewich, 1969.

(2) Temps moyen de germination: TMG $=\frac{\Sigma\left(n_{i} \times t_{i}\right)}{N}$ où $n_{i}$ représente le nombre de graines germées le $t_{j}$ ème jour après semis et $\mathrm{N}$ le nombre total de graines germées. Ce temps est calculé à partir de la date de mise à $5-15^{\circ} \mathrm{C}$ (ne tient donc pas compte des graines prégermées en cours de prétraitement). 
dans la figure 1. Le tableau 1 montre que pour une durée insuffisante du prétraitement (x) des concentrations de 150 à $208 \mathrm{~g}$ de PEG par kilo de solution permettent à la fois un taux de germination élevé, supérieur au témoin, et surtout une germination sensiblement plus rapide. On obtient en effet un gain de 30 à 40 p. 100 sur le temps moyen de germination par rapport au témoin. Ainsi, pour ces deux modalités, la majeure partie des graines germent dans les deux premières semaines ce qui contraste avec le témoin et avec la plus faible concentration en PEG (fig. 1).

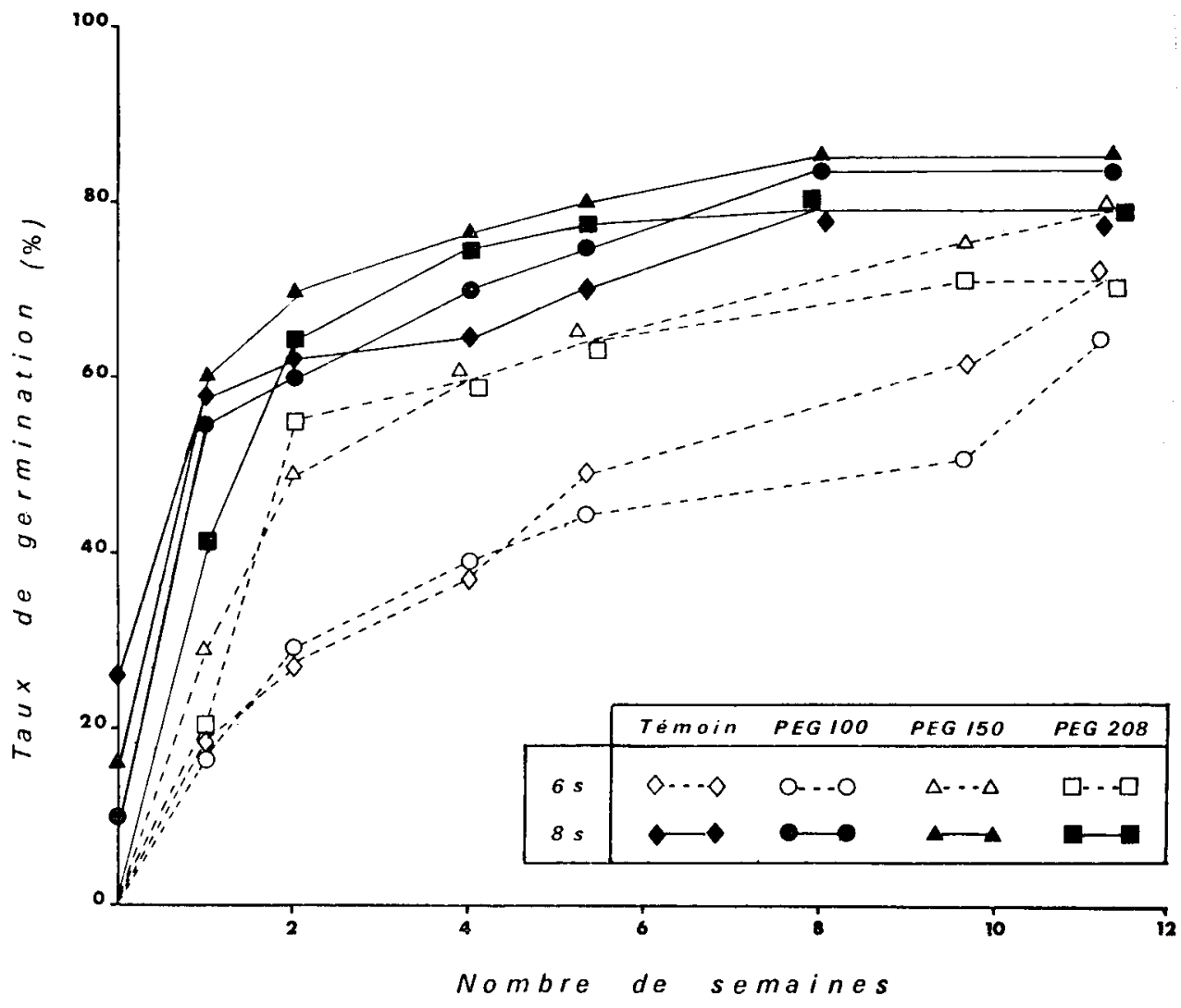

FIG. 1

Courbes de germination d'un lot de faînes

à partir de la mise en températures alternées 5 " $\mathrm{C} / 15^{\circ} \mathrm{C}$ :

Influence de la durée de prétraitement à $+3{ }^{\circ} \mathrm{C}$ (6 semaines [6s] ou 8 semaines [8s]) et de la concentration en polyéthylène glycol (PEG)

exprimée en g par kilo de solution aqueuse.

Germination curves of a lot of beech nuts, starting when seeds are placed in alternate temperatures $\left(5^{\circ} \mathrm{C} / 15^{\circ} \mathrm{C}\right)$ :

the influence of the length of the pretreatment at $3{ }^{\circ} \mathrm{C}$ (6 weeks [6s] or 8 weeks [8s]) and of the concentration of polyethylene glycol (PEG)

expressed in $\mathrm{g}$ per $\mathrm{kg}$ of aqueous solution. 
- Voir si le séchage de faînes ainsi prétraitées est possible, et si elles peuvent ensuite conserver leur état de dormance levée, dans la perspective d'une technique intégrée «levée de dormance - conservation» (SuszKA, 1975; MULLER \& BonNETMASIMBERT, 1981). Bien que suggéré par Heydecker et al., 1975, le séchage des graines traitées au PEG n'est pas toujours possible sans dommage (HEYDECKER, 1977).

- Examiner le comportement en pépinière de faînes ainsi traitées.

- Définir une méthode de prétraitement pour des essais à grande échelle.

Moyennant quoi, cette méthode devrait constituer un outil précieux pour faciliter le remplacement de la technique de stratification par la préréfrigération sans milieu, technique dont nous avons souligné l'intérêt.

Reçu pour publication en octobre 1982.

\author{
Summary \\ Germination improvement of beech nuts through the pretreatment \\ with polyethylene glycol
}

The breaking of dormancy of beech nuts through chilling without any medium seems to be better than the classical stratification in moist peat medium, but it needs a perfect control of the water content of the nuts around 30 p. 100 (fresh weight) during the total length of the treatment. The permanent contact of the nuts with an aqueous solution containing 150 to $200 \mathrm{~g}$ of polyethylene glycol (PEG “6000") per kilo permits this control and prevents any radicle emergence during the cold treatment. Moreover, in the case of an insufficient length of the cold treatment, the PEG treated nuts germinate faster and with a better percentage than do control ones. These preliminary results are encouraging, but further studies are necessary before any practical use of this method.

\title{
Références bibliographiques
}

Duavanshir K., Reid C.P.P., 1975. Effect of moisture stress on germination and radicle development of Pinus eldarica and Pinus ponderosa. Can. J. for. Res., 5, 80-83.

HeYdeCKER W., 1977. Stress and seed germination : an agronomic view. In : The physioNottingham School Agric., 1973/1974, 50-67.

HEYDECKER W., 1977. Stress and seed germination : an agronomic view. In : The physiology and biochemistry of seed dormancy and germination, 237-276, Ed. A.A. Khan, Elsevier/North Holland Biomedical Press.

Heydecker W., Higgins J., Gulliver R.L., 1973. Accelerated germination by osmotic seed treatment. Nature, 246, 42-43.

Heydecker W., Higgins J., Turner Y.J., 1975. Invigoration of seeds? Seed. Sci. Technol., 3, $881-888$.

Kaufmann M.R., 1969. Effects of water potential on germination of lettuce, sunflower and citrus seeds. Can. J. Bot., 47, 1761-1764.

Kaufmann M.R., Ross K.J., 1970. Water potential, temperature and kinetin effects on seed germination in soil and solute systems. Am. J. Bot., 57 (4), 413-419.

LARSON M.M., SchuberT G.H., 1969. Effect of osmotic water stress on germination and initial development of Ponderosa pine seedlings. For. Sci., 15, 30-36. 
MAC Donough W.T., 1976. Water potential of seeds of Bromus inermis and Medicago sativa imbibed on media of various osmotic potentials. Can. J. Bot., 54, 1997-1999.

Muller C., 1977. Dormance et germination des faînes : Bilan des recherches menées en 1976. Doc. Int. Station Amélioration des Arbres forestiers, I.N.R.A., Ardon (France), $\mathrm{n}^{\circ} 77 / 1,32 \mathrm{p}$.

Muller C., 1982. Dormance et germination des faînes : Bilan des recherches menées de

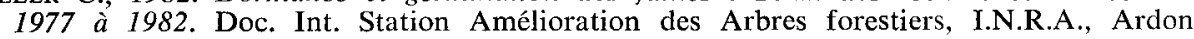
(France), sous presse.

Muller C., Bonnet-Masimbert M., 1981. Technologie de la conservation des faînes. In : Le hêtre, 248-257, Ed. Département des Recherches forestières, I.N.R.A., Paris.

Parmar M.T., Moore R.P., 1968. Carbowax 6000, mannitol and sodium chloride for stimulating drought conditions in germination studies of corn (Zea mays) of strong and weak vigor. Agron. J., 60, 192-195.

Salter P.J., Darby R.J., 1976. Synchronization of germination of celery seeds. Ann. appl. Biol., 84, 415-424.

Satoo T., Goo M., 1954. Seed germination as affected by succion force of soil and saccharose solution. Tokyo Univ. Forest. Bull., 46, 159-168.

SimaK M., 1976. Germination improvement of scots pine seeds from circum polar regions using polyethylene glycol. Proc. 2nd Int. Symp. 1.U.F.R.O., S2.01.06, Japan, 145-153.

Suszka B., 1975. Cold storage of already after-ripened beech (Fagus silvatica L.) seeds. Arbor. Korn., 20, 299-315.

SuszKa B., 1979. Seedling emergence of beech (Fagus silvatica) seed pretreated by chilling without any medium at controlled hydration levels. Arbor. Korn., 24, 111-135.

SuszKa B., ZieTA L., 1976. Further studies on the germination of beech (Fagus silvatica) seed stored in an already after-ripened condition. Arbor. Korn., 21, 279-296.

SuszKa B., Kluczynska A., 1980. Seedling emergence of stored beech (Fagus silvatica L.) seed chilled without medium at a controlled hydration level and pregerminated in cold-moist conditions. Arbor. Korn., 25, 231-255.

Williams J., Shaykewich C.F., 1969. An evaluation of polyethylene glycol (PEG) 6000 and PEG 20,000 in the osmotic control of soil water metric potential. Can. J. Soil. Sci., 49, 397-401. 